\title{
A!
}

This is an electronic reprint of the original article.

This reprint may differ from the original in pagination and typographic detail.

Timofeev, Andrey; Helle, Meri; Meschke, Matthias; Möttönen, Mikko; Pekola, Jukka

\section{Electronic refrigeration at the quantum limit}

Published in:

Physical Review Letters

DOI:

10.1103/PhysRevLett.102.200801

Published: 18/05/2009

Document Version

Publisher's PDF, also known as Version of record

Please cite the original version:

Timofeev, A., Helle, M., Meschke, M., Möttönen, M., \& Pekola, J. (2009). Electronic refrigeration at the quantum limit. Physical Review Letters, 102(20), 1-4. [200801]. https://doi.org/10.1103/PhysRevLett.102.200801

This material is protected by copyright and other intellectual property rights, and duplication or sale of all or part of any of the repository collections is not permitted, except that material may be duplicated by you for your research use or educational purposes in electronic or print form. You must obtain permission for any other use. Electronic or print copies may not be offered, whether for sale or otherwise to anyone who is not an authorised user. 


\title{
Electronic Refrigeration at the Quantum Limit
}

\author{
Andrey V. Timofeev, ${ }^{1,2}$ Meri Helle, ${ }^{1}$ Matthias Meschke, ${ }^{1}$ Mikko Möttönen, ${ }^{1,3,4}$ and Jukka P. Pekola ${ }^{1}$ \\ ${ }^{1}$ Low Temperature Laboratory, Helsinki University of Technology, P.O. Box 3500, 02015 TKK, Finland \\ ${ }^{2}$ Laboratory of Superconductivity, Institute of Solid State Physics, Chernogolovka, 142432 Russia \\ ${ }^{3}$ Department of Applied Physics, Helsinki University of Technology, P.O. Box 5100, 02015 TKK, Finland \\ ${ }^{4}$ Australian Research Council Centre of Excellence for Quantum Computer Technology, The University of New South Wales, \\ Sydney 2052, Australia
}

(Received 14 February 2009; published 18 May 2009)

\begin{abstract}
We demonstrate quantum-limited electronic refrigeration of a metallic island in a low-temperature microcircuit. We show that matching the impedance of the circuit enables refrigeration at a distance, of about $50 \mu \mathrm{m}$ in our case, through superconducting leads with a cooling power determined by the quantum of thermal conductance. In a reference sample with a mismatched circuit this effect is absent. Our results are consistent with the concept of electromagnetic heat transport. We observe and analyze the crossover between electromagnetic and quasiparticle heat flux in a superconductor.
\end{abstract}

DOI: 10.1103/PhysRevLett.102.200801

PACS numbers: 07.20.Mc, 72.15.Eb

The fundamental limit of heat transport via a single channel is governed by the quantum of thermal conductance [1]. This phenomenon was verified experimentally for phonons [2,3], electrons [4], and photons [5]. In the experiment by Meschke et al. [5], the contribution of heat conductance by photons was relatively weak due to impedance mismatch in the employed electrical circuit and due to strong electron-phonon coupling. Here, we demonstrate the importance of matching the circuit to reach the full quantum of heat conductance. Our experiment allows for direct observation of heat transport at the limit of one quantum. We also observe and analyze how two parallel heat conduction mechanisms in a superconductor-by quasiparticles and by thermal photons-dominate in different temperature regimes.

As discovered in 1928 by Johnson and Nyquist [6,7], a resistor $R$ in an electric circuit at temperature $T$ produces thermal voltage noise with power spectrum given by $4 k_{B} T R$ per unit frequency bandwidth. Therefore, two resistors $R_{1}$ and $R_{2}$ at different temperatures $T_{1}$ and $T_{2}$ exchange energy in a circuit with a net heat flux between them: heat flows from hot to cold according to the second law of thermodynamics. The heat flux discussed here is electromagnetic in nature [8], and it can be written as [9]:

$$
P_{\nu}=\int_{0}^{\infty} \frac{d \omega}{2 \pi} \frac{4 R_{1} R_{2} \hbar \omega}{\left|Z_{t}(\omega)\right|^{2}}\left(\frac{1}{e^{\hbar \omega / k_{B} T_{2}}-1}-\frac{1}{e^{\hbar \omega / k_{B} T_{1}}-1}\right)
$$

Here, $Z_{t}(\omega)$ is the frequency $\omega / 2 \pi$ dependent total series impedance of the circuit. Whether the heat exchange in a circuit is classical (as in [6,7]) or quantum-limited depends fundamentally on temperature $T$ and on the (linear) size of the circuit $\ell$, or, more precisely, whether the electromagnetic noise, mediating the heat between the two resistors, is cut-off at the characteristic frequencies of the circuit $\omega_{c}=$ $(R C)^{-1}$ or $\omega_{c}=R / L$, or at the thermal frequency $\omega_{T}=$
$k_{B} T / \hbar$. Rough estimates of unavoidable stray capacitances $C$ and series inductances $L$ are given by $C \sim \epsilon \ell$ and $L \sim$ $\mu \ell$, where $\epsilon$ is the permittivity and $\mu$ the permeability of the medium. For a macroscopic room temperature $T=$ $300 \mathrm{~K}$ circuit of $\ell \sim 1 \mathrm{~mm}$ size, the noise is cutoff at the circuit frequency, $\omega_{c} / \omega_{T} \sim 10^{-2} \ll 1$ for the resistance $R=100 \Omega$, which is of the same order as that in our experiment. In this case the noise and the heat flux are classical, originating from the equipartition law, where each degree of freedom carries an energy $k_{B} T$ on the average [7]. Here, the magnitude of the heat flux does
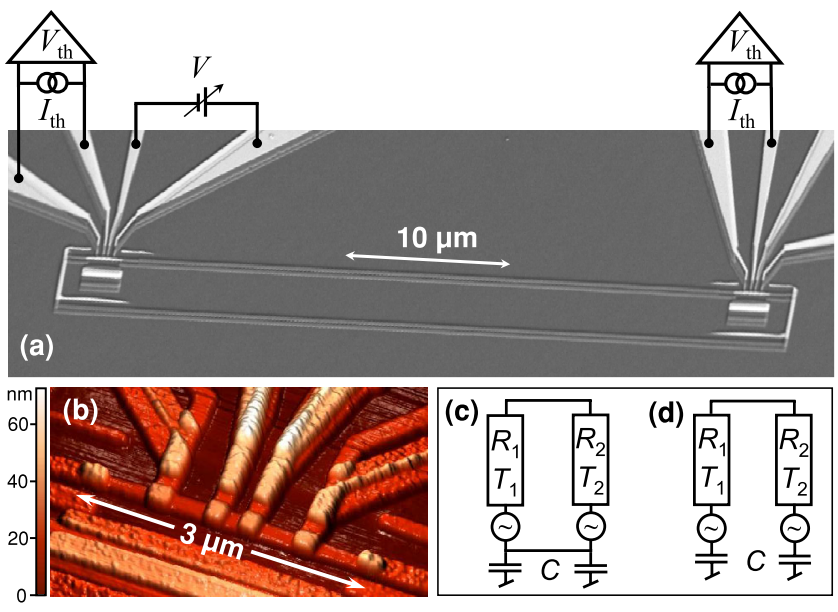

FIG. 1 (color online). (a) Electron micrograph of sample A. Two AuPd islands at a $50 \mu \mathrm{m}$ distance are connected with $\mathrm{Al}$ superconducting lines into a loop to match the impedance between them and enable remote refrigeration. (b) Colored atomic force microscopy image of the island. The four NIS junctions, contacting each island in the middle part, are used to perturb and to measure the island temperature. (c) Equivalent electrical circuit of the matched (sample A) and (d) the mismatched (sample B) structure. 
not follow any universal dependence, but it is determined by the detailed circuit topology and impedances. For a lowtemperature microcircuit as in our experiment, with $T=$ $100 \mathrm{mK}$ and $\ell \sim 100 \mu \mathrm{m}$, we are in the quantum limit: $\omega_{c} / \omega_{T} \sim 10^{2} \gg 1$. In this case, the heat flux is governed by the equilibrium thermal distribution of electromagnetic radiation of the resistor and is limited by the universal quantum of thermal conductance $G_{Q} \equiv \pi k_{B}^{2} T / 6 \hbar$. This electromagnetic heat conduction mechanism dominates in electronic nanostructures [9-12] over electron-phonon and normal electronic heat conduction as the temperature approaches zero.

To observe quantum-limited refrigeration and to demonstrate the significance of impedance matching, we have devised a circuit shown in Fig. 1(a). Two gold-palladium $\left(\mathrm{Au}_{0.75} \mathrm{Pd}_{0.25}\right)$ normal-metal islands on an oxidized silicon substrate at a distance $\ell=50 \mu \mathrm{m}$ are connected into a loop by aluminum $\mathrm{Al}$ superconducting lines. These lines are in a direct contact to the islands, without a tunnel barrier, whereby the contact resistance is small, $<1 \Omega$. Each island is $3 \mu \mathrm{m}$ long, $0.2 \mu \mathrm{m}$ wide, and $20 \mathrm{~nm}$ thick [see Fig. 1(b)] and the measured resistance of each of them is $R \simeq 230 \Omega$. Each island is also connected to four external Al superconducting leads through aluminum oxide tunnel barriers, which form four normal-metal-insulatorsemiconductor (NIS) tunnel junctions, with the area of $150 \times 150 \mathrm{~nm}^{2}$ and measured normal state resistance $R_{T} \simeq 19 \mathrm{k} \Omega$ each. Different pairs of these junctions are used to perturb and to measure the electronic temperature of the islands. We have also fabricated and measured a similar reference sample, in which the two islands were connected only by a single superconducting line and were not enclosed into a loop. The two sample geometries in the experiment represent impedance matched (with loop geometry) and mismatched electrical circuits, which are schematically depicted in Figs. 1(c) and 1(d), and which we denote sample A and sample B, correspondingly.

To show that impedance matching between the two islands is indeed vital for the observation of quantumlimited refrigeration, we compare the rates $P_{\nu}$ in the matched and mismatched cases. For the matched sample A with $R_{1}=R_{2}=R, Z_{t}(\omega)=2 R$, we obtain from Eq. (1) the universal quantum heat flux: $P_{\nu}^{A}=\frac{\pi k_{B}^{2}}{12 \hbar}\left(T_{2}^{2}-T_{1}^{2}\right)$, which is $G_{Q}\left(T_{2}-T_{1}\right)$ for a small temperature difference and presents the maximum heat flux possible for transmission through this electromagnetic channel. For the mismatched circuit with $R_{1}=R_{2}=R$, closed by the capacitance $C / 2 \sim 10 \mathrm{fF}$ of NIS junctions, we obtain $P_{\nu}^{B} \simeq$ $\frac{\pi^{3} k_{B}^{2}}{30 \hbar}\left(T_{2}^{2}-T_{1}^{2}\right)\left(k_{B} T R C / \hbar\right)^{2}$ for $T \approx T_{1} \approx T_{2}$ at high circuit cutoff frequencies $\omega_{c} \gg \omega_{T}$. We find that $P_{\nu}^{B} / P_{\nu}^{A} \simeq \frac{2 \pi^{2}}{5} \times$ $\left(\omega_{T} / \omega_{c}\right)^{2}$; the electromagnetic power flow in the matched circuit is expected to be about $10^{2}-10^{3}$ times stronger as compared to that in the mismatched case at temperatures $0.3-0.1 \mathrm{~K}$ relevant for the experiment.
The two samples were fabricated with the standard methods of electron beam lithography and shadow evaporation, and measured in a ${ }^{3} \mathrm{He}-{ }^{4} \mathrm{He}$ dilution refrigerator as follows. One pair of NIS junctions, the SINIS-refrigerator, connected to island 1, is DC-biased with voltage $V$ to cool down the island by removing hot electrons from it into the superconducting leads through the tunnel barrier at voltages $e V<2 \Delta$ [13-16]. Here $\Delta \simeq 200 \mu \mathrm{eV}$ is the superconducting energy gap of $\mathrm{Al}$ [17]. To probe the island temperature $T_{1}$, the other pair of NIS junctions on the island is used as a thermometer by applying a small DC current $I_{\text {th }}$ through it, and by measuring the corresponding temperature dependent voltage $V_{\text {th }}$. Another similar SINISthermometer probes the temperature $T_{2}$ of the second island. When the applied voltage $V$ through the SINISrefrigerator is zero, the measured voltage $V_{\text {th }}(V=0)$ provides the thermometer calibration against the cryostat bath temperature $T_{0}$ varied in the range $50-500 \mathrm{mK}$. The electronic temperature of the islands is then obtained from the fit of the dependence of $T_{0}$ on $V_{\mathrm{th}}(V=0)$. At $V=0$ the electronic temperature coincides with the bath temperature down to $T_{0}=120 \mathrm{mK}$. The thermometers have individual floating DC bias sources and do not cause excessive heating or cooling of the islands due to the low bias current, $I_{\text {th }} \simeq 0.001 \Delta / e R_{T}$, used.

The thermal model that accounts for our setup and observations is shown in Fig. 2. The resistors exchange energy at power $P_{\nu}$ through the electromagnetic channel, and, in parallel, at power $P_{s}$, due to quasiparticle heat conduction through the superconducting line. The latter contribution is significant at higher temperatures but diminishes exponentially towards low-temperatures, $k_{B} T_{0} \ll \Delta[18,19]$. We analyze quasiparticle heat flux with the heat diffusion equation

$$
\frac{d}{d x}\left(-\kappa_{s} \frac{d T}{d x}\right)=\alpha\left(T_{0}\right) \Sigma_{\mathrm{Al}}\left[T_{0}^{5}-T^{5}(x)\right]
$$

assuming that the superconducting line has the temperature profile $T(x)$ with boundary conditions $T(0)=T_{2}$ and $T(\ell)=T_{1}$, where $x$ is the coordinate along the line

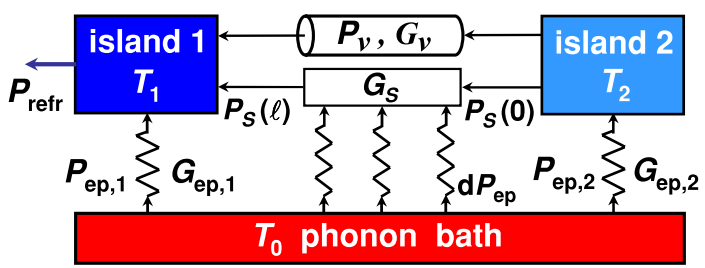

FIG. 2 (color online). The thermal model illustrates electromagnetic $\left(P_{\nu}, G_{\nu}\right)$ and quasiparticle $\left(P_{s}, G_{s}\right)$ heat conduction through the superconducting line between the two islands. The islands are thermally coupled to the phonon bath with the heat fluxes $P_{\text {ep, } 1}$ and $P_{\text {ep }, 2}$. The weak electron-phonon coupling of the superconducting line to the thermal bath is denoted by $d P_{\text {ep }}$. The arrows show the direction of the heat flow for temperatures $T_{1}<$ $T_{2}<T_{0}$. 
( $x=0$ corresponds to the contact to island $2, x=\ell$ to that to island 1). Factor $\alpha\left(T_{0}\right)$ determines the suppression of electron-phonon coupling in the superconducting line with respect to that in the normal-metal state [18], and $\Sigma_{\mathrm{Al}} \simeq$ $0.3 \times 10^{9} \mathrm{~W} \mathrm{~K}^{-5} \mathrm{~m}^{-3}$ is a material constant for aluminum [16]. The heat flux $P_{s}(0)=-\kappa_{s} A T^{\prime}(0)$ to island 1 , and the heat flux $P_{s}(L)=-\kappa_{s} A T^{\prime}(\ell)$ from island 2 are determined through temperature gradients $T^{\prime}(0)$ and $T^{\prime}(\ell)$ at the ends of the superconducting line with cross-sectional area $A=$ $200 \times 25 \mathrm{~nm}^{2}$. Here, $\kappa_{s}=\gamma\left(T_{0}\right) \kappa_{n}$ is the heat conductivity of the superconducting line [19], suppressed by a factor $\gamma\left(T_{0}\right)=\frac{3}{2 \pi^{2}} \int_{\Delta\left(T_{0}\right) / k_{B} T_{0}}^{\infty} \frac{t^{2} d t}{\cosh ^{2}(t / 2)}$ with respect to heat conductivity $\kappa_{n}=\ell L_{0} T(x) /\left(R_{\ell} A\right)$ in the normal state determined by the Wiedemann-Franz law. Here, $L_{0} \simeq$ $2.4 \times 10^{-8} \mathrm{~W} \Omega \mathrm{K}^{-2}$ is the Lorenz number, and $R_{\ell}$ is the normal state resistance of the aluminum line; $R_{\ell} \simeq 138 \Omega$ for sample $\mathrm{A}$ and $R_{\ell} \simeq 188 \Omega$ for sample $\mathrm{B}$. The electrons in each resistor of volume $\Omega_{i}$ exchange energy with the substrate, i.e., with the thermal bath at temperature $T_{0}$ via electron-phonon coupling at the rate $P_{\mathrm{ep}, i}=$ $\Sigma_{\mathrm{AuPd}} \Omega_{i}\left(T_{0}^{5}-T_{i}^{5}\right)$ [20-22], where $\Sigma_{\mathrm{AuPd}} \simeq(3 \pm 1) \times$ $10^{9} \mathrm{~W} \mathrm{~K}^{-5} \mathrm{~m}^{-3}$ is obtained from the measurements. Island 1 can be cooled (or heated) with power $P_{\text {refr }}$. We neglect phonon heat transport based on experimental results discussed below. The steady state of the system is then described by the energy balance equations

$$
\begin{array}{r}
P_{\text {refr }}-P_{\nu}-P_{s}(\ell)-P_{\mathrm{ep}, 1}=0, \\
P_{\nu}+P_{s}(0)-P_{\mathrm{ep}, 2}=0 .
\end{array}
$$

We solve numerically Eq. (2) together with Eqs. (3) to obtain the relative temperature change of island 2 with respect to that of island $1, \Delta T_{2} / \Delta T_{1} \equiv\left(T_{2}-T_{0}\right) /\left(T_{1}-\right.$ $\left.T_{0}\right)$. For small temperature differences, neglecting the electron-phonon coupling in the superconductor, we can linearize the different contributions in Eqs. (3) and obtain a particularly simple expression for $\Delta T_{2} / \Delta T_{1}$ :

$$
\frac{\Delta T_{2}}{\Delta T_{1}}=\frac{G_{\nu}+G_{s}}{G_{\nu}+G_{s}+G_{\mathrm{ep}, 2}} .
$$

Here, the photon coupling $G_{\nu}$ is expected to be equal to $G_{Q}$ for the matched sample and for the mismatched sample it is suppressed by a large factor as discussed above. The electron-phonon conductance is given by $G_{\mathrm{ep}, 2}=$ $5 \Sigma_{\text {AuPd }} \Omega_{2} T_{0}^{4}$, and $G_{s}$ denotes quasiparticle heat conductance in the superconducting line.

Upon sweeping the voltage $V$ across the SINISrefrigerator, both thermometers show cooling at $\mathrm{eV} \lesssim$ $2 \Delta$, indicating electronic refrigeration of both islands; see Figs. 3(a) and 3(b). As expected, the SINIS refrigerating effect is maximal around the optimal bias voltage $e V \simeq$ $2 \Delta$. The absolute temperature drops $\Delta T_{1}$ and $\Delta T_{2}$ of the two islands for the two samples are shown in Figs. 3(c) and 3(d). In both cases, the direct SINIS refrigeration of island 1 is similar. In contrast, the refrigeration of the remote
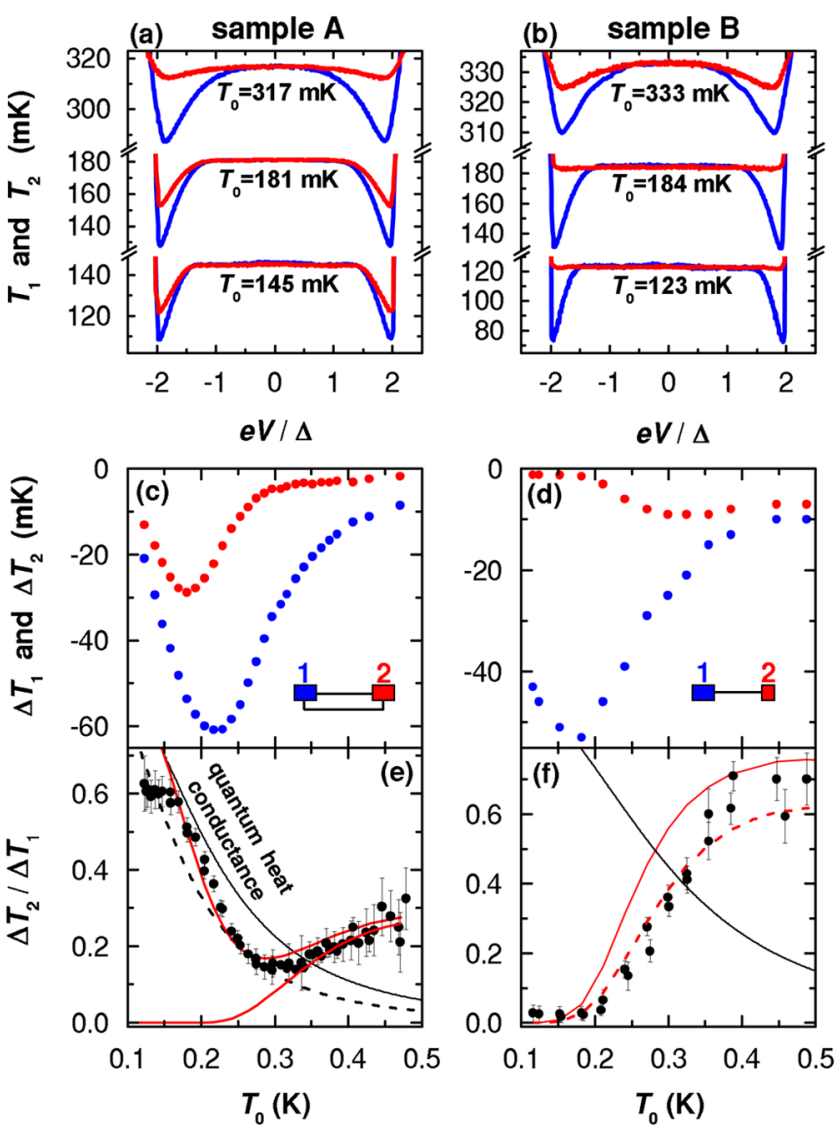

FIG. 3 (color online). Measured data of sample A (a),(c),(e) and sample B (b),(d),(f) and results from the thermal model. (a), (b) Measured island temperatures $T_{1}$ (blue line) and $T_{2}$ (red line) vs bias voltage $V$ at three bath temperatures $T_{0}$. (c),(d) Absolute temperature changes $\Delta T_{1}$ (blue dots) and $\Delta T_{2}$ (red dots) measured at $T_{0}=120-500 \mathrm{mK}$. (e),(f) Relative temperature changes at $T_{0}=120-500 \mathrm{mK}$ (dots). The error bars show the standard deviation arising from the temperature calibration. (e), (f) The black lines are obtained from the linearized thermal model. The red lines are the results of the numerical thermal model. The solid lines are calculated with $\Sigma_{\text {AuPd }}=$ $2 \times 10^{9} \mathrm{~W} \mathrm{~K}^{-5} \mathrm{~m}^{-3}$ and the dashed lines with $\Sigma_{\mathrm{AuPd}}=$ $4 \times 10^{9} \mathrm{~W} \mathrm{~K}^{-5} \mathrm{~m}^{-3}$

island is drastically different in the two samples. In sample A with the matched circuit, island 2 temperature tends to follow the temperature of the main island at lowtemperatures. In sample B with the mismatched circuit, the refrigeration of the remote island is suppressed at lowtemperatures. The corresponding relative temperature drops $\Delta T_{2} / \Delta T_{1}$ against $T_{0}$ at the optimum cooling bias voltage are plotted in Figs. 3(e) and 3(f). For the matched sample $\Delta T_{2} / \Delta T_{1}$ has a minimum as a function of $T_{0}$ at about $300 \mathrm{mK}$; below this temperature it increases rapidly, as can be expected based on strong electromagnetic coupling. In the mismatched sample, $\Delta T_{2} / \Delta T_{1}$ vanishes towards lower temperatures, due to weak photonic coupling.

For the matched sample, the rise of $\Delta T_{2} / \Delta T_{1}$ at lowtemperatures in Fig. 3(e) is in agreement with the simple 
linearized thermal model: the data below $300 \mathrm{mK}$ lie between the solid and dashed black lines, obtained from Eq. (4) assuming full quantum conductance $G_{\nu}=G_{Q}$ and vanishing quasiparticle conductance $G_{s}=0$. Alternatively, the dashed black curve can be obtained from Eq. (4) with $G_{\nu}=0.5 G_{Q}$ and $\Sigma_{\mathrm{AuPd}}=2 \times$ $10^{9} \mathrm{~W} \mathrm{~K}^{-5} \mathrm{~m}^{-3}$, since only the photonic $G_{\nu}$ and the electron-phonon $G_{\mathrm{ep}, 2}$ coupling contribute to the relative temperature drop $\Delta T_{2} / \Delta T_{1}$ when $G_{s}=0$. Based on these results we conclude that the matching in sample $\mathrm{A}$ is close to ideal and the refrigeration is limited by the quantum of thermal conductance. This effect is absent in the mismatched sample: for reference we show the black line in Fig. 3(f) with $G_{\nu}=G_{Q}$ and $G_{s}=0$.

The quantitative behavior of $\Delta T_{2} / \Delta T_{1}$ at high temperatures $T_{0} \geqslant 300 \mathrm{mK}$ is not universal and depends on sample parameters. In this temperature regime, the remote island of sample B is refrigerated more than that in sample A. This is because of stronger thermalization of sample A with larger island 2, and since there are, due to the deposition technique, extra normal (AuPd) shadows covering the vertical parts of the Al looped line [see Fig. 1(a)]. In sample $\mathrm{B}$, the normal shadow is not in contact with the superconducting line, which further enhances the quasiparticle mediated refrigeration. For sample A, the data over the full temperature range are accounted for by the upper red line in Fig. 3(e), obtained from the numerical analysis with $P_{\nu}=P_{\nu}^{A}$ and $\Sigma_{\mathrm{AuPd}}=2 \times 10^{9} \mathrm{~W} \mathrm{~K}^{-5} \mathrm{~m}^{-3}$. To fit the data for sample $\mathrm{A}$ in the diffusion regime at temperatures above $300 \mathrm{mK}$, we added a fitting parameter $\alpha_{N}=0.6$ to the factor $\alpha: \alpha \rightarrow \alpha+\alpha_{N}$. The parameter $\alpha_{N}$ describes stronger thermalization of the superconducting line. The lower red line in Fig. 3(e), calculated with $\alpha_{N}=0.6$ and with no photonic heat exchange $P_{\nu}=0$, shows quasiparticle contribution for comparison. For sample B, the numerically obtained red curves of quasiparticle conduction are shown in Fig. 3(f). The dashed red curve shows good agreement with the data. The uncertainty in the quantitative comparison between the model and data arises from only approximately known parameters of electron-phonon coupling for AuPd and $\mathrm{Al}$ thin films.

In both samples, at voltages $e V>2 \Delta$, the probe islands are strongly heated due to hot quasiparticle injection. An additional thermometer, located near island 2, but not connected to it, was monitoring phonon temperature on the substrate. It showed negligibly weak temperature response as compared to the thermometers of islands 1 and 2. This supports our thermal model, which assumes that phonons provide a good thermal bath and that the observed heat exchange between the resistors occurs due to quasiparticles and electromagnetic coupling. This is a natural conclusion due to the very weak electron-phonon coupling at low temperatures.
To summarize, we have demonstrated quantum-limited refrigeration: the low-temperature data show quantitative agreement with the thermal model assuming heat conduction determined by the quantum of thermal conductance. Furthermore, our observations and model account for residual heat conduction in a superconductor by quasiparticles. We suggest that even galvanically decoupled resistors can be refrigerated by the mechanism discussed. This could be an option for noise suppression purposes in sensitive quantum devices, e.g., by refrigerating shunt resistors [21] in SQUIDs.

We thank N. Chekurov for technical assistance in AFM imaging. This work is supported by the Academy of Finland, National Graduate School for Materials Physics, and the NanoSciERA project "NanoFridge" of the EU.

[1] J. B. Pendry, J. Phys. A 16, 2161 (1983).

[2] K. Schwab et al., Nature (London) 404, 974 (2000).

[3] C.S. Yung, D. R. Schmidt, and A. N. Cleland, Appl. Phys. Lett. 81, 31 (2002).

[4] O. Chiatti et al., Phys. Rev. Lett. 97, 056601 (2006).

[5] M. Meschke, W. Guichard, and J.P. Pekola, Nature (London) 444, 187 (2006).

[6] J. B. Johnson, Phys. Rev. 32, 97 (1928).

[7] H. Nyquist, Phys. Rev. 32, 110 (1928).

[8] There are generally other contributions of heat flux, which are often dominant, like that between the resistor and the phonon bath, i.e., the usual conduction to the surroundings, and electronic heat conduction along the wiring.

[9] D. R. Schmidt, R. J. Schoelkopf, and A. N. Cleland, Phys. Rev. Lett. 93, 045901 (2004).

[10] T. Ojanen and T. T. Heikkilä, Phys. Rev. B 76, 073414 (2007).

[11] T. Ojanen and A. P. Jauho, Phys. Rev. Lett. 100, 155902 (2008).

[12] D. Segal, Phys. Rev. Lett. 100, 105901 (2008).

[13] M. Nahum, T. M. Eiles, and J. M. Martinis, Appl. Phys. Lett. 65, 3123 (1994).

[14] M. M. Leivo, J. P. Pekola, and D. V. Averin, Appl. Phys. Lett. 68, 1996 (1996).

[15] A. M. Clark et al., Appl. Phys. Lett. 86, 173508 (2005).

[16] F. Giazotto et al., Rev. Mod. Phys. 78, 217 (2006).

[17] The measured value of $\Delta$ is $200 \pm 5 \mu \mathrm{eV}$ for sample A and $210 \pm 5 \mu \mathrm{eV}$ for sample B.

[18] A. V. Timofeev et al., Phys. Rev. Lett. 102, 017003 (2009).

[19] J. Bardeen, G. Rickayzen, and L. Tewordt, Phys. Rev. 113, 982 (1959).

[20] M. L. Roukes et al., Phys. Rev. Lett. 55, 422 (1985).

[21] F. C. Wellstood, C. Urbina, and J. Clarke, Phys. Rev. B 49, 5942 (1994).

[22] The volume $\Omega_{2}$ of island 2 for sample $A$ is $1.2 \times$ $10^{-20} \mathrm{~m}^{3}$ and for sample B it is $4.3 \times 10^{-21} \mathrm{~m}^{3}$. 